\title{
Compact IR Quadrupoles for Linear Colliders Based on Rutherford-type Cable
}

\author{
M. L. Lopes, V. S. Kashikhin, V. V. Kashikhin, A.V. Zlobin
}

\begin{abstract}
The upcoming and disrupted beams in the interaction region (IR) of a linear collider are focused by doublets consisting of two small-aperture superconducting quadrupoles. These magnets need an effective compact magnetic shielding to minimize magnetic coupling between the two channels and sufficient temperature margin to withstand radiation-induced heat depositions in the coil. This paper presents conceptual designs of IR quadrupoles for linear colliders based on NbTi and $\mathrm{Nb}_{3} \mathrm{Sn}$ Rutherford-type cables.
\end{abstract}

Index Terms-Accelerator magnet, Rutherford-type cable, Superconducting quadrupole, Linear collider.

\section{INTRODUCTION}

$\mathrm{C}$ Tompact FD doublets placed on each side of the ILC detector focus the upcoming electron and positron beams at the Interaction Point (IP). Each doublet consists of two small-aperture superconducting (SC) quadrupoles QD0 and QF1 and multipole correctors (see Fig.1). The two outgoing disrupted beams are channeled into extraction beam lines by two larger aperture SC quadrupoles QDEX1 and QFEX2 [1]. QD0 with its corrector and QDEX1 are placed in the same cryostat and installed inside the detector. The effect of the detector solenoid field on the incoming beams will be mitigated by a special solenoid with opposite field direction installed around QD0 and QDEX1 in the same cryostat [2]. The other two quadrupoles, QF1 with corrector and QFEX2, are placed in a separate cryostat outside the detector. It is expected that ILC IR magnet cooling be provided by superfluid helium at $2 \mathrm{~K}$.

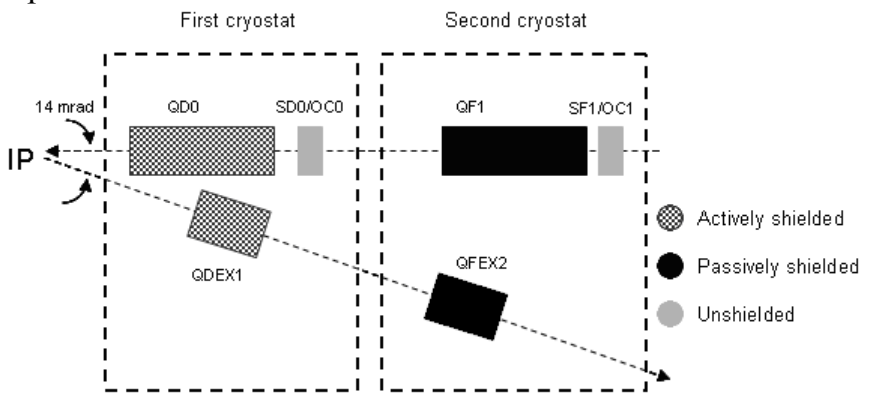

Fig. 1. ILC IR layout [1] (not in scale).

Manuscript received August 25, 2008. This work was supported by Fermi Research Alliance, LLC, under contract No. DE-AC02-07CH11359 with the U.S. Department of Energy.

M. L. Lopes, V. S. Kashikhin, V. V. Kashikhin, A. V. Zlobin are with the Fermi National Accelerator Laboratory, Batavia, IL 60510 USA (corresponding author phone: 630-840-2003; fax: 630-840-3369; e-mail: mllopes@fnal.gov).
The present baseline design of ILC IR quadrupoles is based on a $\mathrm{NbTi}$ round cable and direct wind technology. The quadrupoles QD0 and QDEX1 closest to the IP use active magnetic shields provided by external quadrupole coils. The other two quadrupoles can use passive iron shields. The magnet design and technology have been demonstrated by a series of short prototypes [3].

This approach has some attractive features, but it is not free from some disadvantages. The multilayer multi-turn coil design increases the magnet inductance and limits the accuracy of turn position control, complicates coil azimuthal pre-load, limits radial and azimuthal heat transfer, etc. Thermal analysis of IR quadrupoles with such low radial and azimuthal thermal conductivity in the ILC IR radiation environment showed that QFEX2 based on the baseline design has insufficient operation margin [4]. In addition, the direct wind technology was demonstrated at the present time only for $\mathrm{NbTi}$ superconductor.

This paper describes conceptual designs of compact IR quadrupoles based on shell-type coils and Rutherford-type cables which are suitable for linear colliders. This approach is being widely used in accelerator magnets. It meets all the requirements and is free from the mentioned above limitations. Moreover, it is compatible with both $\mathrm{NbTi}$ and $\mathrm{Nb}_{3} \mathrm{Sn}$ magnet technologies.

\section{Magnetic Design AND PARAMETERS}

The main design parameters of ILC IR quadrupoles can be found on the web [5]. They are summarized in Table I.

This paper focuses on QD0 and QDEX1 since these magnets have the most challenging parameters and requirements to their design. First of all, due to the proximity of QDEX1 and QD0, these magnets have to be shielded to minimize the magnetic coupling between them. However, the location of these magnets inside the detector solenoid and the limited radial space exclude the use of iron yoke for fringe field shielding. Active shielding is used instead. The solenoidal field is also added to the self field of the coil, which reduces the operation margin, and needs to be taken into consideration.

TABLE I DESIGN PARAMETERS OF ILC IR QUADRUPOLES

\begin{tabular}{lcccc}
\hline \hline & QDO & QF1 & QDEX1 & QFEX2 \\
\hline Aperture, mm & 28 & 28 & 38 & 60 \\
Nominal gradient, T/m & 142 & 80 & 100 & 23 \\
Magnetic length, m & 2.20 & 2.00 & 1.06 & 1.20 \\
\hline \hline
\end{tabular}



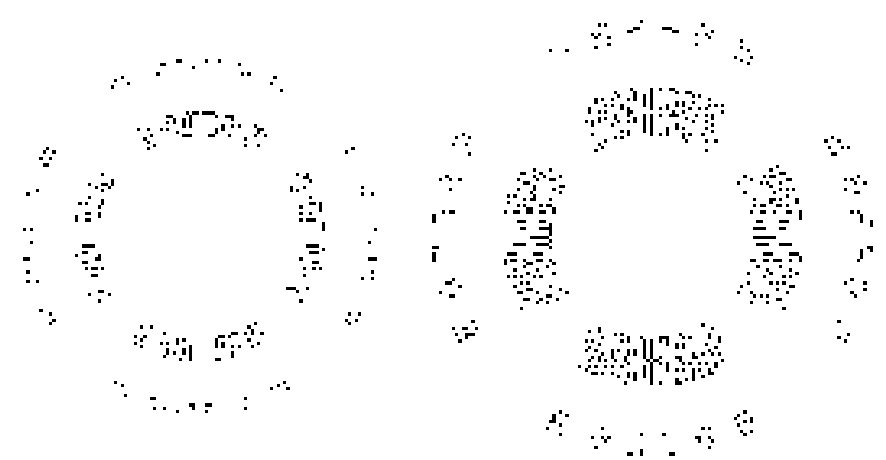

Fig. 2. Single (left) and double (right) layers quadrupole designs. The outer layer is the active shield.

Figure 2 shows the two compact designs of quadrupole coils with active magnetic shield for the ILC interaction region. The innermost single or double layer represents the main coil. The active shielding is provided by the outer single or double layer coil. Table II summarizes the design and performance parameters of these quadrupoles.

\section{TABLE II QUADRUPOLE DESIGN PARAMETERS}

\begin{tabular}{lcccc}
\hline \hline & \multicolumn{2}{c}{ QD0 } & \multicolumn{2}{c}{ QDEX1 } \\
\hline Coil design & $\begin{array}{c}\text { Single } \\
\text { layer }\end{array}$ & $\begin{array}{c}\text { Double } \\
\text { layer }\end{array}$ & $\begin{array}{c}\text { Single } \\
\text { layer }\end{array}$ & $\begin{array}{c}\text { Double } \\
\text { layer }\end{array}$ \\
Superconductor type & $\mathrm{Nb}_{3} \mathrm{Sn}$ & $\mathrm{NbTi}$ & $\mathrm{Nb}_{3} \mathrm{Sn}$ & $\mathrm{NbTi}$ \\
Operation temperature, $\mathrm{K}$ & 4.2 & 1.9 & 4.2 & 1.9 \\
Coil ID, mm & & 28 & & 38 \\
Magnet OD, mm & 49.4 & 61 & 61.4 & 76.8 \\
Coil cross-section, mm ${ }^{2}$ & 37.1 & 81.9 & 52.1 & 97.1 \\
$\mathrm{~B}_{\text {peak }}$ (magnet body), $\mathrm{T}$ & 5.84 & 4.86 & 6.0 & 5.0 \\
$\mathrm{I}_{\text {max }} @ \mathrm{~B}_{\text {peak }}, \mathrm{A}$ & 9971 & 4728 & 9617 & 4578 \\
$\mathrm{G}_{\text {max }}, \mathrm{T} / \mathrm{m}$ & 302.7 & 284.5 & 226.2 & 215.6 \\
$\mathrm{G}_{\text {max }} / \mathrm{G}_{\text {nom }}$ & 2.13 & 2.00 & 2.26 & 2.16 \\
Inductance, $\mathrm{mH} / \mathrm{m}_{\mathrm{Stored}_{\text {energy at }}}$ & 0.18 & 0.89 & 0.24 & 1.17 \\
\hline \hline
\end{tabular}

The geometrical parameters of the cables used in the main and shielding coils are reported in Table III. The NbTi strand considered in this study had $\mathrm{J}_{\mathrm{c}}(5 \mathrm{~T}, 4.2 \mathrm{~K})=2500 \mathrm{~A} / \mathrm{mm}^{2}$ and a $\mathrm{Cu} / \mathrm{Sc}$ ratio of 1.3. The $\mathrm{Nb}_{3} \mathrm{Sn}$ strand had $\mathrm{J}_{\mathrm{c}}(12 \mathrm{~T}, 4.2 \mathrm{~K})=2500 \mathrm{~A} / \mathrm{mm}^{2}$ and a $\mathrm{Cu} / \mathrm{Sc}$ ratio of 0.85 . These are standard parameters for commercially available strands.

TABLE III CABLE PARAMETERS

\begin{tabular}{lcc}
\hline \hline & Main coil & Shielding coil \\
\hline Number of strands & 12 & 6 \\
Strand diameter, mm & 0.5 & 0.7 \\
Cable width, mm & 3.066 & 2.123 \\
Average thickness, mm & 0.893 & 1.249 \\
Insulation thickness, mm & 0.1 & 0.1 \\
\hline \hline
\end{tabular}

TABLE IV QD0 BASELINE DESIGN PARAMETERS

\begin{tabular}{lc}
\hline \hline Nominal current, A & 779 \\
Stored energy at the nominal gradient, $\mathrm{kJ} / \mathrm{m}$ & 1.62 \\
Inductance, $\mathrm{mH} / \mathrm{m}$ & 5.3 \\
Coil cross-section, $\mathrm{mm}^{2}$ & 42.6 \\
Magnet OD, $\mathrm{mm}$ & 70 \\
\hline \hline
\end{tabular}

The operation margin defined as $\mathrm{G}_{\max } / \mathrm{G}_{\text {nom }}$ is more than 2 for all the designs presented in Table II. The single-layer $\mathrm{Nb}_{3} \mathrm{Sn}$ coil designs reduce the magnet OD by $20 \%$ and can operate at higher temperatures with a simpler and more effective cryogenic system.

Table IV summarizes the parameters simulated for the baseline ILC quadrupole design QD0 described in [2, 3, 5]. As can be seen from Tables II and IV, the OD of the baseline design is larger than that of the proposed double-layer and single-layer designs based on $\mathrm{NbTi}$ and $\mathrm{Nb}_{3} \mathrm{Sn}$ cables respectively. The stored energy in the baseline design is smaller than in the designs presented in this paper. However, the inductance of the proposed designs is much smaller than that of the baseline magnet. This is an important factor when considering the magnet quench protection system.

The excess of maximum field gradient provided by these new quadrupoles can be translated in terms of their design margin. The design margin will take into account not only the usual problems during magnet construction, but also the external solenoidal field and the heat deposition due to the disrupted beam.

The magnet coils were also optimized for the field quality. The design target was to zero the $b_{6}$ and $b_{10}$ geometrical harmonics at a reference radius equal to half of the quadrupole's aperture. The field quality was evaluated considering RMS errors of 50 microns for the radial and azimuthal positioning of the coil blocks. Table $\mathrm{V}$ summarizes the results for QD0. As can be seen, the standard deviations for all the harmonics are quite high due to the fact that the assumed geometrical errors are large compared to the transverse dimensions of these magnets. The effect of the expected field quality in the described magnets on the beam parameters needs to be carefully analyzed.

TABLE V FIELD HARMONICS ERRORS (QD0)

\begin{tabular}{ccccc}
\hline \hline & \multicolumn{3}{c}{$\mathrm{a}_{\mathrm{n}}$} & \multicolumn{2}{c}{$\mathrm{b}_{\mathrm{n}}$} \\
\cline { 2 - 5 } $\mathrm{n}$ & mean & sigma & mean & sigma \\
\hline 3 & -0.63 & 9.33 & 0.08 & 9.89 \\
4 & -0.24 & 5.48 & -0.34 & 6.03 \\
5 & -0.15 & 3.12 & 0.04 & 2.92 \\
6 & 0.02 & 1.58 & 0.03 & 1.62 \\
7 & 0.02 & 0.79 & 0.02 & 0.81 \\
8 & -0.01 & 0.40 & -0.03 & 0.39 \\
9 & 0.00 & 0.19 & 0.00 & 0.18 \\
10 & 0.00 & 0.09 & 0.00 & 0.09 \\
\hline \hline
\end{tabular}




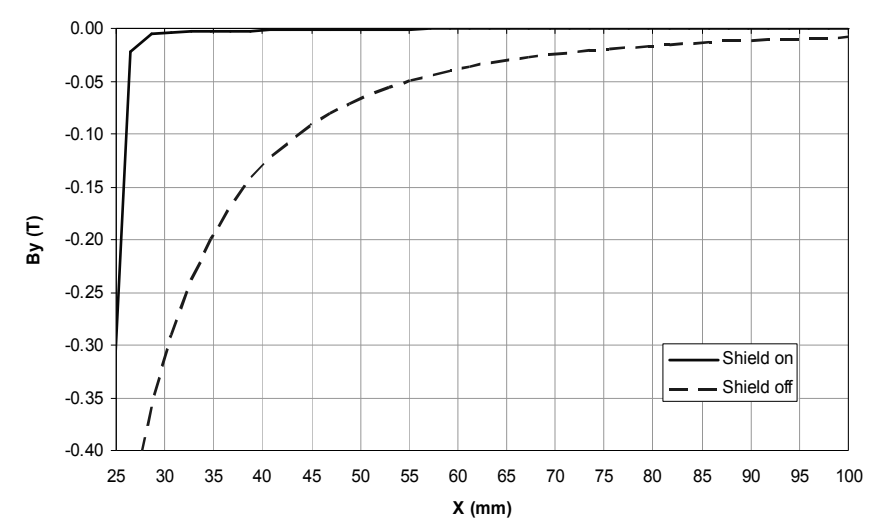

Fig. 3. The fringe field produced by QD0 at nominal gradient with active shield "on" and "off". X=0 corresponds to QD0 center.

\section{ACTIVE ShIELD OptimizATION}

QD0 and QDEX1 designs utilize active shielding coils around the main coils to reduce the magnetic coupling of the incoming and disrupted beam lines. The main and shielding coils are connected in series and powered from one power supply. The geometry of shielding coils in all the previously described designs was optimized to achieve the required field compensation outside the magnet and reduce the magnet transverse size. The active shield reduces the quadrupole strength of the main coil by about $19 \%$. Figure 3 shows the effect of active shielding for the single layer QD0 quadrupole. Considering a minimum separation of $77 \mathrm{~mm}$ between QD0 and QDEX1, the QD0 shielding coil reduces the field on the QDEX1 axis from $18 \mathrm{mT}$ (shield "off") to $0.2 \mathrm{mT}$ (shield "on").

Figure 4 shows the distribution of the vertical field component $\mathrm{B}_{\mathrm{y}}$ produced by QD0 along the QDEX1 axis $\mathrm{Z}$ (at $\mathrm{x}=77 \mathrm{~mm}$ ) with active shield for the three relative lengths of QD0 main and shielding coils. As can be seen, the level of field compensation significantly changes along the magnet axis near the coil ends. The integrated shielding effect depends on the lengths of the main and shielding coils.
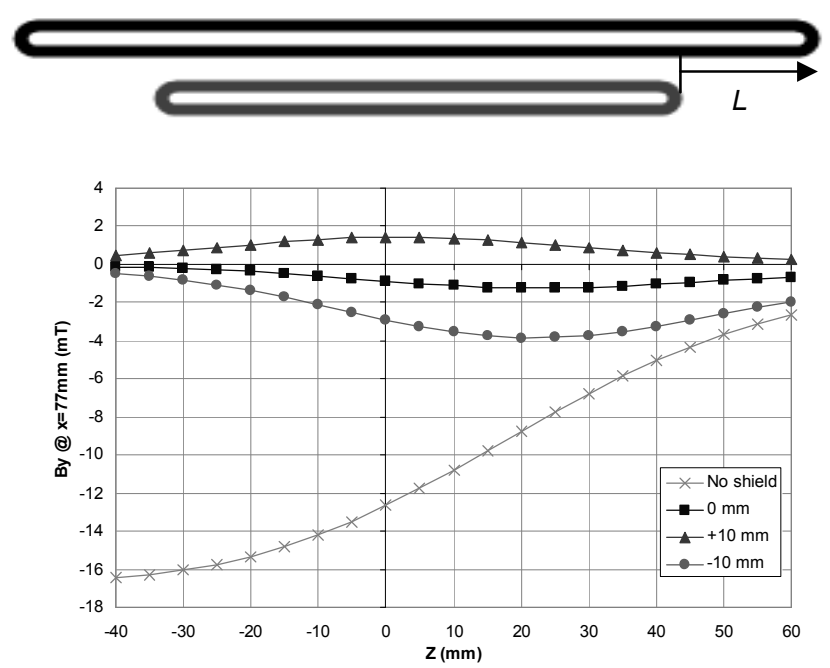

Fig. 4. Distribution of the vertical field component $\mathrm{B}_{\mathrm{y}}$ produced by QD0 along QDEX1 axis (z). $\mathrm{Z}=0$ corresponds to the end of the main QD0 coil. $\mathrm{L}$ is the difference between the main coil and shielding coil end position.

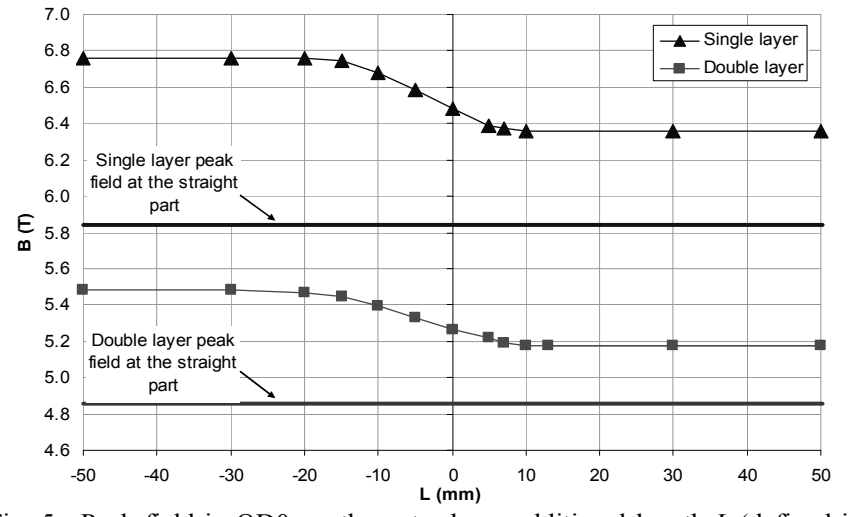

Fig. 5. Peak field in QD0 vs. the outer layer additional length $L$ (defined in Fig.4) for the single-layer $\mathrm{Nb}_{3} \mathrm{Sn}$ design and the double-layer $\mathrm{NbTi}$ design.

The average fringe field (the field integrated along the axis and normalized by the magnet length) on QDEX1 axis from QD0 without shielding reaches $22 \mathrm{mT}$. With shielding for the same lengths of the main and shielding coils $(\mathrm{L}=0)$ it reduces to $0.7 \mathrm{mT}$. As can be seen from the data in Figure 4, it can be further reduced by slightly increasing the shielding coil length (the optimum is between 0 and $+10 \mathrm{~mm}$ ).

It is well known that the peak field in unshielded superconducting magnets is located in the coil ends. Figure 5 shows the peak field in the coil ends as function of the shielding coil additional length $\mathrm{L}$ for the single-layer $\mathrm{Nb}_{3} \mathrm{Sn}$ design and the double-layer NbTi design. The horizontal lines represent the peak field in the magnet straight section for both designs. The peak field in the coil ends is always higher than the peak field in the magnet body. With the optimal shielding coil length $(\mathrm{L} \sim+5 \mathrm{~mm})$ the end peak field reduces by $\sim 5 \%$ for both designs. The high peak field in coil ends reduces the maximum gradient by $4 \%$ with the optimal shield length for both double and single layer designs.

\section{DETECTOR SOLENOID EFFECT}

QD0 and QDEX1 quadrupoles will be placed in a cryostat inside the detector solenoid. The external field will reduce the safety margin on these magnets. Figure 6 shows the reduction of the maximum gradient as function of the external fields. As can be seen, a reduction of the maximum gradient up to $22 \%$ could be observed in the external field up to $3 \mathrm{~T}$.

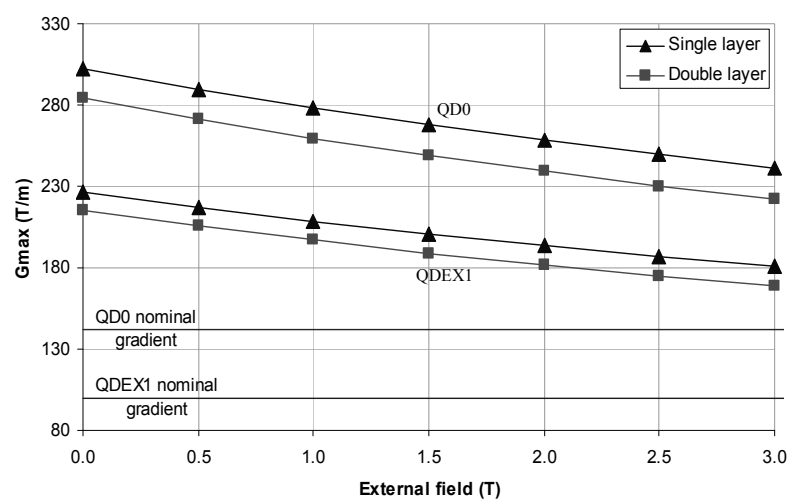

Fig. 6. Maximum gradient vs. the external field for the single-layer $\mathrm{Nb}_{3} \mathrm{Sn}$ quadrupole operating at $4.2 \mathrm{~K}$ and for the double-layer NbTi quadrupole operating at $1.9 \mathrm{~K}$. 
The field produced by the detector solenoid in the area of focusing quadrupoles needs to be reduced in order to minimize its negative effect on the incoming beam. This is planned to be done with the help of a short anti-solenoid placed around the QD0 and QDEX1. The anti-solenoid will also increase the operation margin of focusing quadrupoles by reducing the detector solenoid contribution to the maximum field in the quadrupole coils.

\section{TEMPERATURE MARGIN}

Using the critical surface parameterizations for $\mathrm{NbTi}$ and $\mathrm{Nb}_{3} \mathrm{Sn}$ superconductors [6]-[8], the magnet temperature margins were calculated for QD0 and QDEX1 based on both single-layer and double-layer designs, $\mathrm{NbTi}$ and $\mathrm{Nb}_{3} \mathrm{Sn}$ coils, for two possible operation temperatures, $1.9 \mathrm{~K}$ and $4.2 \mathrm{~K}$. Figures 7 and 8 show the magnet temperature margin with respect to the operation temperature as function of the maximum gradient for QD0 and QDEX1, respectively. As can be seen, the single-layer $\mathrm{Nb}_{3} \mathrm{Sn}$ quadrupoles can operate at both temperatures with an operation margin more than 2 . The double-layer $\mathrm{NbTi}$ quadrupoles can also operate at both temperatures; however, the operation margin at $4.2 \mathrm{~K}$ reduces to 1.5 for QD0 and 1.6 for QDEX1. It may not be sufficient for the reliable operation of these magnets in accelerator.

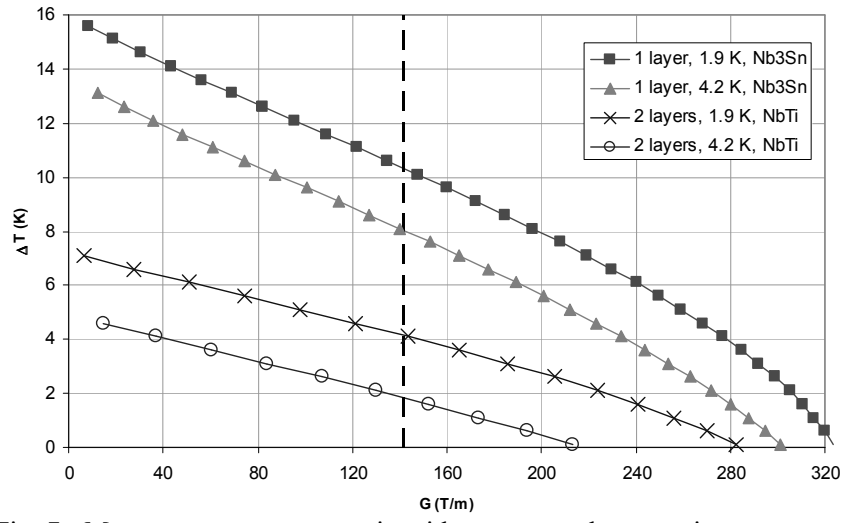

Fig. 7. Magnet temperature margin with respect to the operation temperature as function of the field gradient for QD0. The vertical dashed line represents the nominal QD0 gradient.

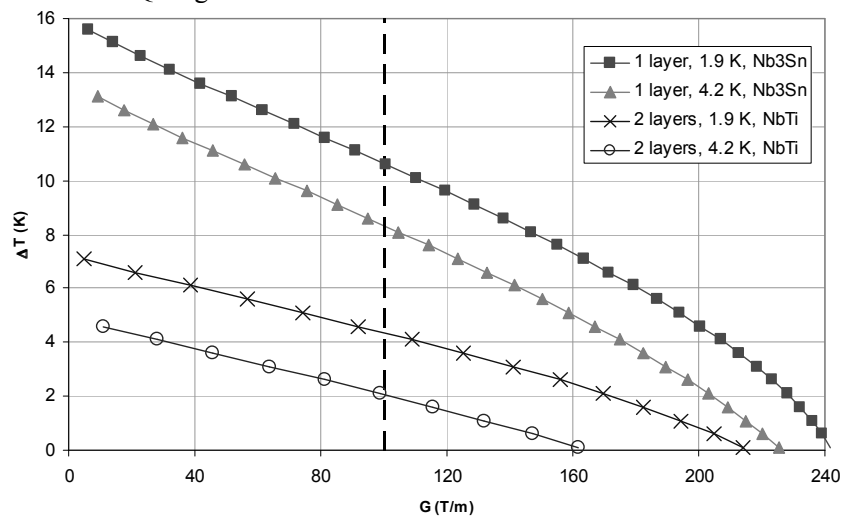

Fig. 8. Magnet temperature margin with respect to the operation temperature as function of the field gradient for QDEX1. The vertical dashed line represents the nominal QDEX1 gradient.

\section{CONCLUSION}

Conceptual designs of ILC IR quadrupoles QD0 and QDEX1 based on Rutherford cable and active shield have been developed and studied. Magnet design parameters meet the ILC IR specifications.

It was shown that the design approach based on Rutherford cable has several advantages. Coils built with the Rutherford cable have a high packing factor which allows higher efficiency and more compact magnet design. The good turn positioning in the coils, the turn radial and azimuthal pre-load and support during operation provide stable field quality and alignment in current and thermal cycles during long-term operation. Low coil inductance helps to protect the magnet during a quench. Due to the self support of turns in the coils based on Rutherford cable, the beam pipe and the coil are thermally decoupled, which reduces coil heating from the heat deposited in the beam pipe. High radial thermal conductivity increases the magnet operation margin with respect to the radiation heat deposition in the coil.

The described design approach is compatible with both $\mathrm{NbTi}$ and $\mathrm{Nb}_{3} \mathrm{Sn}$ superconductors presently used in accelerator magnets. In particular, the use of $\mathrm{Nb}_{3} \mathrm{Sn}$ cable with higher critical temperature, critical field and current density allows reducing the coil volume by $15 \%$, the magnet size by $40 \%$ and opens the possibility of operating the IR magnets at $4.2 \mathrm{~K}$.

The effect of an active shield length on $\mathrm{B}_{\max }$ in the coil and the QD0 fringe field variation along the QDEX1 axis were studied. The optimal length of active shield coil with respect to the main coil was determined.

\section{REFERENCES}

[1] A. Seryi et al., "Design of the Beam Delivery System for the International Linear Collider", Proc. of PAC07, Albuquerque, NM, USA, June 2007.

[2] B. Parker et al, "The Superconducting Magnets of the ILC Beam Delivery System", Proc. of PAC07, Albuquerque, NM, USA, June 2007.

[3] B. Parker et al, "Compact Superconducting Final Focus Magnet Options for the ILC", Proc. of PAC 2005, Knoxville, TN, USA, June 2005.

[4] A. Drozhdin, et al., "Radiation and Thermal Analysis of Final Doublet Quadrupoles in the Interaction Region of Linear Collider", Proc. of EPAC'2008, Genoa, Italy, June 2008.

[5] http://www.linearcollider.org/cms/?pid=1000437

[6] M.S. Lubell, "Empirical scaling formulas for critical current and critical fields for commercial NbTi”, IEEE Trans. Magnetics, Vol.19, No. 3, 1983, pp.754-757.

[7] A. Devred, CEA/Saclay, "Review of Superconducting Dipole and Quadrupole Magnets for Particle Accelerators", DAPNIA/STCM 98-07, August 1998.

[8] L.T. Summers et al., "A model for the prediction of $\mathrm{Nb}_{3} \mathrm{Sn}$ critical current as a function of field, temperature, strain, and radiation damage", IEEE Trans. Magnetics, Vol. 27, No. 2, Mar 1991, pp. 2041-2044. 